\section{Kidney \\ Blood Pressure \\ Research}

\title{
Lisinopril Protects Against the Adriamycin Nephropathy and Reverses the Renalase Reduction: Potential Role of Renalase in Adriamycin Nephropathy
}

\author{
Pengxun Han ${ }^{\mathrm{a}, \mathrm{c}}$ Huili Sun ${ }^{\mathrm{a}, \mathrm{c}}$ Yuanzhao Xua Youjia Zeng ${ }^{\mathrm{a}}$ Wuyong Yia \\ Jia Wua Mumin Shao ${ }^{\mathrm{b}}$ Shunmin Lia Tiegang Yia
}

aDepartment of Nephrology, Shenzhen Affiliated Hospital, Guangzhou University of Chinese Medicine, Shenzhen, China; 'bepartment of Pathology, Shenzhen Affiliated Hospital, Guangzhou University of Chinese Medicine, Shenzhen, China; 'Pengxun Han and Huili Sun contributed equally to this work

\section{Key Words}

Renalase • Adriamycin nephropathy $•$ Blood pressure $•$ Lisinopril

\begin{abstract}
Aims: To investigate the potential role of renalase in adriamycin nephropathy and the effect of lisinopril on the regulation of renalase. Methods: Adriamycin nephropathy was induced in male Wistar rats $(n=12)$ by a single injection of adriamycin at $2 \mathrm{mg} / \mathrm{kg}$ body weight. Rats were then randomly assigned to a model group or a treatment group, to which were administered distilled water or the angiotensin converting enzyme inhibitor lisinopril, respectively, for 12 weeks. Six normal rats served as controls. At the end of study, physiological parameters and systolic blood pressure were measured. Glomerulosclerosis and tubulointerstitial injury were assessed by histopathology. Renalase protein expression in kidney was quantified by immunohistochemistry and immunoblotting. The serum concentration and urinary excretion of renalase were determined by enzyme-linked immunosorbent assay. Results: In model group rats, proteinuria and systolic blood pressure were elevated. Increased serum renalase concentration was observed; however, renalase protein expression in the kidney was significantly decreased. Compared with the model group, decreased proteinuria, lower systolic blood pressure, and fewer morphologic lesions were detected in the treatment group. Although levels of serum renalase were similar, accumulation of renalase in urine and kidney tissue increased notably in the treatment group compared with the model group. Conclusions: This study suggests that renalase may be involved in the process of adriamycin-induced renal injuries. Lisinopril may attenuate adriamycin-induced kidney injury by controlling blood pressure, which may be partially attributed to the renalase expression and secretion.
\end{abstract}




\section{Kidney Blood Pressure Research}

\section{Introduction}

Renalase is a novel flavin adenine dinucleotide-dependent amine oxidase, which was first discovered by Gary V. Desir in 2005 [1]. It has been shown that renalase can metabolize circulating catecholamines and regulate blood pressure. Renalase consists of 342 amino acids, has a molecular weight of approximately $37.8 \mathrm{kDa}$, and is primarily distributed in the kidney, heart, skeletal muscle, and small intestine [1, 2]. In renal tissue, renalase is primarily detected in renal tubular epithelial cells, mesangial cells and podocytes. A previous in vitro study of renal cells showed that only tubular epithelial cells secreted renalase into the supernatant, suggesting that these cells are the primary renalase-secreting cells in kidney [3]. It was also reported that renalase could be activated and secreted into the blood by infusion of catecholamines and/or a transient increase in blood pressure. When renalase was activated, it degraded plasma catecholamines, and thus caused a significant fall in blood pressure [4].

A case-control study in a northern Han Chinese population revealed that genetic variations in the renalase gene may influence susceptibility to essential hypertension, and the gene was thus identified as a novel susceptibility gene for essential hypertension [5]. A functional mis-sense polymorphism in renalase (Glu37 Asp) is associated with cardiovascular diseases such as cardiac hypertrophy, ventricular dysfunction, poor exercise capacity, and inducible ischemia in persons with stable coronary artery disease [6]. Renalase deficiency in a renalase knockout (KO) mouse model is associated with increased plasma catecholamine levels, blood pressure and susceptibility to ischemic myocardial damage. Furthermore, renalase replacement therapy may ameliorate cardiovascular complications [7].

Another study demonstrated that subcutaneous infusion of recombinant human renalase into 5/6 nephrectomy model rats caused a decrease in cardiac contractility, heart rate, and systolic blood pressure (SBP), which might be explained by its ability to metabolize catecholamines [8]. Taken together, these data suggest that renalase plays an important role in the regulation of blood pressure and sympathetic tone, particularly associated with chronic kidney disease (CKD). Despite this, its physiological function is at present ambiguous. Renalase may serve as a novel target on the treatment of hypertension and sympathetic over-activity, especially in kidney diseases. Blockade of the renin-angiotensin system (RAS) is an effective therapeutic strategy in kidney diseases [9]. In addition to beneficial effects on proteinuria, blood pressure, and amelioration of morphologic lesions in kidney, inhibition of RAS is capable of reducing sympathetic overactivity[10]. While previous studies [11-15] demonstrated that ACEI could reduce proteinuria, lower SBP, ameliorate tubulointerstitial injury and slow the progression of glomerulosclerosis in adriamycin nephropathy rats, the relationship between RAS and renalase in this model was unclear.

In the present study, we investigated changes of renalase in adriamycin nephropathy and confirmed that ACEI may play a renoprotective role by regulating renalase expression and secretion in this model.

\section{Materials and Methods}

\section{Animal model}

Male Wistar rats $(\mathrm{n}=18)$ weighing 170 220g were purchased from the Laboratory Animal Unit of Southern Medical University (Guangzhou, China). All experiments were conducted in accordance with the NIH statements of the "Principles of laboratory animal care". Rats were treated according to the guidelines of the Institutional Animal Care and Use Committee of the Guangzhou University of Traditional Chinese Medicine. The animals were housed at a constant temperature $\left(21^{\circ} \mathrm{C}\right)$ under a controlled $12 \mathrm{~h}$ light:dark cycle and had free access to water and standard laboratory diet. Experimental adriamycin nephropathy rats $(n=12)$ were induced by a single injection via the tail vein $(2 \mathrm{mg} / \mathrm{kg}$ body weight) of adriamycin (Sigma Aldrich, St. Louis, MI, USA) in sterile water. The rats were then randomly allocated into either a model group 


\section{Kidney \\ Blood Pressure Research}

Kidney Blood Press Res 2013;37:295-304

\begin{tabular}{l|l}
\hline DOI: $10.1159 / 000350157$ & (C) 2013 S. Karger AG, Basel
\end{tabular}

Published onlıne: September 05, 2013

www.karger.com/kbr

Han/Sun/Xu/Zeng/Yi/Wu/Shao/Li/Yi: Renalase in Adriamycin Nephropathy

(AN group, $n=6$ ) or a treatment group (AN+LIS group, $n=6$ ). Model group rats were administered with distilled water. Treatment group rats were administered with lisinopril (LIS, Cayman Chemicals, Catalog No.16833, Ann Arbor, MI, USA) at a dose of $10 \mathrm{mg} / \mathrm{kg}$ body weight per day. Treatments were administered intragastrically for 12 weeks from the onset of adriamycin nephropathy. Six normal rats served as control (control group, $\mathrm{n}=6$ ) and were administered with distilled water.

\section{Physiological and metabolic parameters}

Body weight was measured weekly and rats were placed in metabolic cages (Tecniplast S.p.a, Buguggiate, Italy) every 4 weeks for $24 \mathrm{~h}$ urine collection. Several drops of toluene were added to the urine collection beaker to inhibit microbial growth. Total protein in urine was measured by turbidimetry and creatinine was measured by an enzymatic colorimetric method using an automated biochemical analyzer (Roche, Basel, Switzerland). Prior to the study, rats were trained to become accustomed to handling, and to SBP measurements. At 12 weeks, SBP was assessed by tail plethysmography in conscious animals in a preheated plethysmograph chamber using a blood pressure measurement system (IITC Life Science, Woodland Hills, CA, USA) as previously described [16]. Three measurements were taken over 5 minutes and the mean value was calculated. At the end of the study, blood samples were taken by cannulation of the abdominal aorta. Serum albumin, total protein, creatinine, urea, triglycerides, and cholesterol were all measured using the Roche automated biochemical analyzer.

\section{Tissue preparation}

At the end of the study (12 weeks after lisinopril administration) all rats were anesthetized by intraperitoneal injection with pentobarbital sodium (Sigma-Aldrich, St. Louis, MI, USA) at a dose of $50 \mathrm{mg}$ / kg body weight. A midline incision of the abdomen was cut and blood samples were obtained from the aorta. The kidneys were removed immediately, weighed and rinsed in phosphate buffer solution (PBS). Sections of the kidneys were stored in $10 \%$ formalin for subsequent histopathological examination and immunohistochemical studies. The remaining renal tissues were immediately snap-frozen in liquid nitrogen and stored at $-80^{\circ} \mathrm{C}$ for later analysis.

\section{Kidney histopathology}

Paraffin sections were stained with periodic acid-Schiff to evaluate the glomerulosclerosis index (GSI) and tubulointerstitial injury (TII). GSI was assessed in 50 randomly selected glomeruli per kidney at objective magnification of 40x, and graded using a semi-quantitative scoring method as previously described [17]. Glomerulosclerosis is defined as mesangial matrix expansion, capillary obliteration, hyalinosis, and adhesion of the glomerular tuft to Bowman's capsule. If the glomerulus was normal, a score of 0 was given; if $0 \sim 25 \%$ was affected, a score of 1 was given; $25 \sim 50 \%$ was scored as 2; 50 75\% was scored as 3 and $75 \sim 100 \%$ was scored as 4 . TII refers to tubular cast, atrophy, dilatation, and presence of inflammatory cells. TII was assessed in 10 randomly sampled fields per kidney at objective magnification of $10 \times$ and the changes were graded as follows: grade 0 , normal; grade 1 , the area of interstitial inflammation, tubular atrophy, and dilation with cast formation involving $<25 \%$ of the field; grade 2, lesion area between $25 \%$ and $50 \%$ of the field; and grade 3 , lesions involving $>50 \%$ of the field. The indices for TII were calculated by averaging the grades assigned to all fields of tubules [16].

\section{Immunohistochemistry}

At 12 weeks, immunohistological examinations were performed. In brief, formalin-fixed kidney sections ( $4 \mu \mathrm{m}$ thick) taken from rats in each group were mounted on slides, dewaxed and hydrated. Slides were brought to the boil in $10 \mathrm{mM}$ sodium citrate buffer (pH 6) for $10 \mathrm{~min}$ and cooled on a bench top for 30 min. After 10 minutes incubation in 3\% hydrogen peroxide, sections were blocked with normal goat serum for $30 \mathrm{~min}$, and then stained overnight at $4^{\circ} \mathrm{C}$ with renalase-specific primary antibodies (ab109013, 1:200, Abcam, Cambridge, UK). After washing with rinse buffer, sections were incubated with $50 \mu \mathrm{L}$ amplifier (Reagent A, Elivision ${ }^{\mathrm{TM}}$ super HRP (Mouse/Rabbit) IHC Kit, Maixin-Bio, Fuzhou, China) for 10 min at room temperature. The sections were then washed with rinse buffer and incubated with HRP-Polymer Conjugated anti-Mouse/Rabbit IgG complex (Reagent B, Elivision ${ }^{\mathrm{TM}}$ super HRP (Mouse/Rabbit) IHC Kit, Maixin-Bio, Fuzhou, China) for $10 \mathrm{~min}$ at room temperature. Localization of peroxidase conjugates was determined using diaminobenzidine tetrahydrochloride solution as chromogen and haematoxylin as counterstain. 


\section{Kidney \\ Blood Pressure Research}

For evaluation of the immunoperoxidase stain of renalase, 10 fields per kidney at objective magnification of $20 \times$ were randomly selected from renal cortex and medulla respectively. The immunoperoxidase stain was quantified using NIS-Elements imaging software Version 4.10 (Nikon Corporation, Tokyo, Japan). Renalase expression level was calculated by multiplication of the positive area and mean optical density.

\section{Western blot}

Renalase protein expression in the kidney was quantified by western blotting, and alpha-tubulin was also quantified in the same samples as a control. Snap-frozen kidneys were homogenized in lysis buffer of the following composition: $20 \mathrm{mM}$ Tris- $\mathrm{HCl}$ (pH 7.5), $150 \mathrm{mM} \mathrm{NaCl}, 1 \mathrm{mM} \mathrm{Na}{ }_{2}$ EDTA, $1 \mathrm{mM}$ EGTA, 1\% NP-40, $1 \%$ sodium deoxycholate, $2.5 \mathrm{mM}$ sodium pyrophosphate, $1 \mathrm{mM} \beta$-glycerophosphate, $1 \mathrm{mM} \mathrm{Na}_{3} \mathrm{VO}_{4}, 1 \mu \mathrm{g} / \mathrm{ml}$ leupeptin and $1 \mathrm{mM}$ PMSF. The homogenate was centrifuged at $14000 \times \mathrm{g}$ for $15 \mathrm{~min}$ at $4^{\circ} \mathrm{C}$ and supernatant protein concentrations were measured by BCA protein assay kit (Pierce Biotechnology, Rockford, IL, USA). A total of $60 \mu \mathrm{g}$ protein of each sample was separated on a $10 \%$ sodium dodecylsulfate-polyacrylamide gel, and then transferred to a nitrocellulose membrane (Bio-Rad Laboratories, Hercules, CA, USA). Nonspecific binding sites of the membrane were blocked at room temperature for $1 \mathrm{~h}$ with $0.5 \mathrm{~g} / \mathrm{l} \mathrm{non-fat} \mathrm{milk} \mathrm{power} \mathrm{in}$ Tris-buffered saline/Tween-20, and then incubated overnight at $4{ }^{\circ} \mathrm{C}$ with primary antibodies: rabbit antirenalase antibody (ab109013, 1:1000, Abcam, Cambridge, UK), mouse anti- $\alpha$-tubulin antibody (1:1000, Cell Signaling Technologies, MA, USA). The membrane was then incubated with secondary antibodies: horseradish peroxidase-conjugated anti-rabbit IgG (1:1000, Cell Signaling Technologies, MA, USA) or antimouse IgG (1:1000, Cell Signaling Technologies, MA, USA) for $1 \mathrm{~h}$ at room temperature with shaking. Blots were visualized using an enhanced chemiluminescence detection system (Pierce Biotechnology, Rockford, IL, USA). For quantitative evaluation of the western blot studies, the films were scanned and the optical densities were quantified using VisionWorks LS Imagine Acquisition and Analysis Software (UVP Inc, Upland, CA, USA). The results were presented as density values of renalase as a ratio of $\alpha$-tubulin density values.

\section{Enzyme-linked immunosorbent assay (ELISA)}

Serum and urine renalase concentrations were determined by ELISA using the method described by Rong Gu [18]. The serum and urine was collected after centrifugation at $1620 \times \mathrm{g}$ at $4^{\circ} \mathrm{C}$ for $15 \mathrm{~min}$, and then stored at $-80^{\circ} \mathrm{C}$ until analysis. 96 -well plates were coated with renalase peptide (Abcam, Cambridge, UK) at a concentration of $0.5 \mu \mathrm{g} / \mathrm{ml}$, diluted in bicarbonate buffer ( $\mathrm{pH} 9.6$ ), and incubated at $4{ }^{\circ} \mathrm{C}$ overnight. After blocking with $1 \%$ bovine serum albumin (Sigma-Aldrich, St. Louis, MI, USA) at room temperature for $1 \mathrm{~h}, 50$ $\mu \mathrm{L}$ serum or urine samples and $50 \mu \mathrm{L}$ anti-renalase antibody (1:4000, Abcam, Cambridge, UK) were added to the coated wells and incubated at $37^{\circ} \mathrm{C}$ for $2 \mathrm{~h}$. After washing with PBST, horseradish peroxidase (HRP)conjugated rabbit polyclonal antibody to goat IgG (1:5000, Abcam, Cambridge, UK) was used as secondary antibody. After the termination of coloration after 30 minutes (by the addition of $2 \mathrm{~mol} / \mathrm{L} \mathrm{H}_{2} \mathrm{SO}_{4}$ ), OD at 450 $\mathrm{nm}$ was measured using an iMark $^{\mathrm{TM}}$ microplate absorbance reader (Bio-Rad, Hercules, CA, USA).

\section{Statistics}

Data are expressed as mean \pm SD. Between-group differences were analyzed by one-way ANOVA with correction for multiple comparisons using the Least-Significant Difference post-hoc test for multiple comparisons. Statistical analyses were performed using SPSS statistical software, version 16.0. A $P$ value of less than 0.05 was considered statistically significant.

\section{Results}

Physiological and metabolic parameters

Compared to control rats, increased proteinuria $(74.45 \pm 22.90 \mathrm{mg} / 24 \mathrm{~h})$ was observed in response to adriamycin administration at 4 weeks, and this gradually increased with time. In the treatment group, lisinopril partially prevented the adriamycin-induced increase in proteinuria (Table 1). At 12 weeks, hypoalbuminemia and hyperlipidemia were detected in model group rats, whereas these conditions were alleviated by lisinopril (Table 2). Model group rats had higher SBP than control rats at 12 weeks $(159.67 \pm 10.97 \mathrm{mmHg}$ vs. 


\section{Kidney \\ Blood Pressure Research}

Table 1. Time course of urinary protein excretion $(\mathrm{mg} / 24 \mathrm{~h})$ after injection of adriamycin

\begin{tabular}{cccc}
\hline Weeks & 4 & 8 & 12 \\
\hline Control & $16.50 \pm 2.33$ & $14.53 \pm 2.50$ & $14.03 \pm 4.16$ \\
AN & $74.45 \pm 22.90^{\mathrm{a}}$ & $141.96 \pm 48.45^{\mathrm{a}}$ & $144.20 \pm 25.74^{\mathrm{a}}$ \\
AN+LIS & $26.45 \pm 16.33^{\mathrm{b}}$ & $64.81 \pm 41.93^{\mathrm{b}}$ & $70.63 \pm 47.08^{\mathrm{b}}$ \\
\hline \multicolumn{4}{l}{} \\
\hline
\end{tabular}

Table 2. Physiological and biochemical parameters in various groups of animals at 12 weeks

\begin{tabular}{cccc}
\hline Parameter & Control & AN & AN+LIS \\
\hline $\mathrm{N}$ & 6 & 6 & 6 \\
Body weight $(\mathrm{g})$ & $432.67 \pm 22.03$ & $382.50 \pm 25.74^{\mathrm{a}}$ & $381.17 \pm 30.35^{\mathrm{a}}$ \\
Left kidney (g) & $1.16 \pm 0.10$ & $1.28 \pm 0.16$ & $1.12 \pm 0.11$ \\
Left kidney/body & $2.68 \pm 0.14$ & $3.34 \pm 0.39^{\mathrm{a}}$ & $2.95 \pm 0.20^{\mathrm{b}}$ \\
weight (mg/g) & & & \\
SBP (mmHg) & $124.22 \pm 12.38$ & $159.67 \pm 10.97^{\mathrm{a}}$ & $112.17 \pm 9.20^{\mathrm{b}}$ \\
ALB (g/L) & $35.68 \pm 0.72$ & $28.23 \pm 3.30^{\mathrm{a}}$ & $31.85 \pm 2.09^{\mathrm{b}}$ \\
TP (g/L) & $54.25 \pm 1.67$ & $52.50 \pm 2.79$ & $53.10 \pm 2.39$ \\
TG (mmol/L) & $1.48 \pm 0.45$ & $2.93 \pm 2.07^{\mathrm{a}}$ & $1.57 \pm 0.51^{\mathrm{b}}$ \\
CHOL (mmol/L) & $1.75 \pm 0.19$ & $3.91 \pm 1.71^{\mathrm{a}}$ & $2.57 \pm 0.54^{\mathrm{b}}$ \\
Serum creatinine & $35.93 \pm 5.87$ & $37.95 \pm 2.49$ & $38.55 \pm 4.46$ \\
$(\mu \mathrm{mol} / \mathrm{L})$ & $8.80 \pm 0.40$ & $8.20 \pm 0.71$ & $8.28 \pm 0.92$ \\
BUN (mmol/L) & &
\end{tabular}

Data are shown as mean $\pm \mathrm{SD}$, a $P<0.05$ vs. control rats, $\mathrm{b} P<0.05$ vs. AN rats.

$124.22 \pm 12.38 \mathrm{mmHg}$ ). Compared to the model group, SBP in the treatment group rats was significantly reduced by lisinopril. No differences in serum creatinine or BUN were observed among the three groups at 12 weeks. Compared to control rats, model group rats had slower weight gain during the experimental period and increased left kidney/body weight ratio at 12 weeks. This ratio in the treatment group was much lower than in the model group, and only slightly higher than in control group (Table 2).

Glomerulosclerosis index (GSI) and tubulointerstitial injury (TII)

Twelve weeks after adriamycin administration, model group rats displayed focal segmental sclerotic changes in the glomeruli, characterized by collapse and obliteration of glomerular tufts. Hyalin deposition in the glomerular tufts and adhesion of the tufts to Bowman's capsule were also clearly observed (Figure 1B). In the tubulointerstitial region of model rats, tubular casts, atrophy and interstitial inflammatory cells were also observed (Figure 1E). Compared with the model group, lisinopril treatment significantly ameliorated these lesions (Figure 1C, F). Quantitative analysis of GSI and TII were shown in Figure 1G and $\mathrm{H}$.

Renalase protein expression in the renal tissue

In control group rats, renalase was mainly distributed in the proximal tubules of the kidney, with lower expression in glomeruli. As shown in Figure 2 (A, B, D, E), in the model 


\section{Kidney Blood Pressure Research}

Fig. 1. Periodic acid-Schiff staining of formalin-fixed kidney sections taken from representative rats in each group $\quad(n=6$ rats/group) at 12 weeks. The reddish-purple color indicates the presence of glycogen or polysaccharide deposits. Results shown are representative images from different kidney samples from each

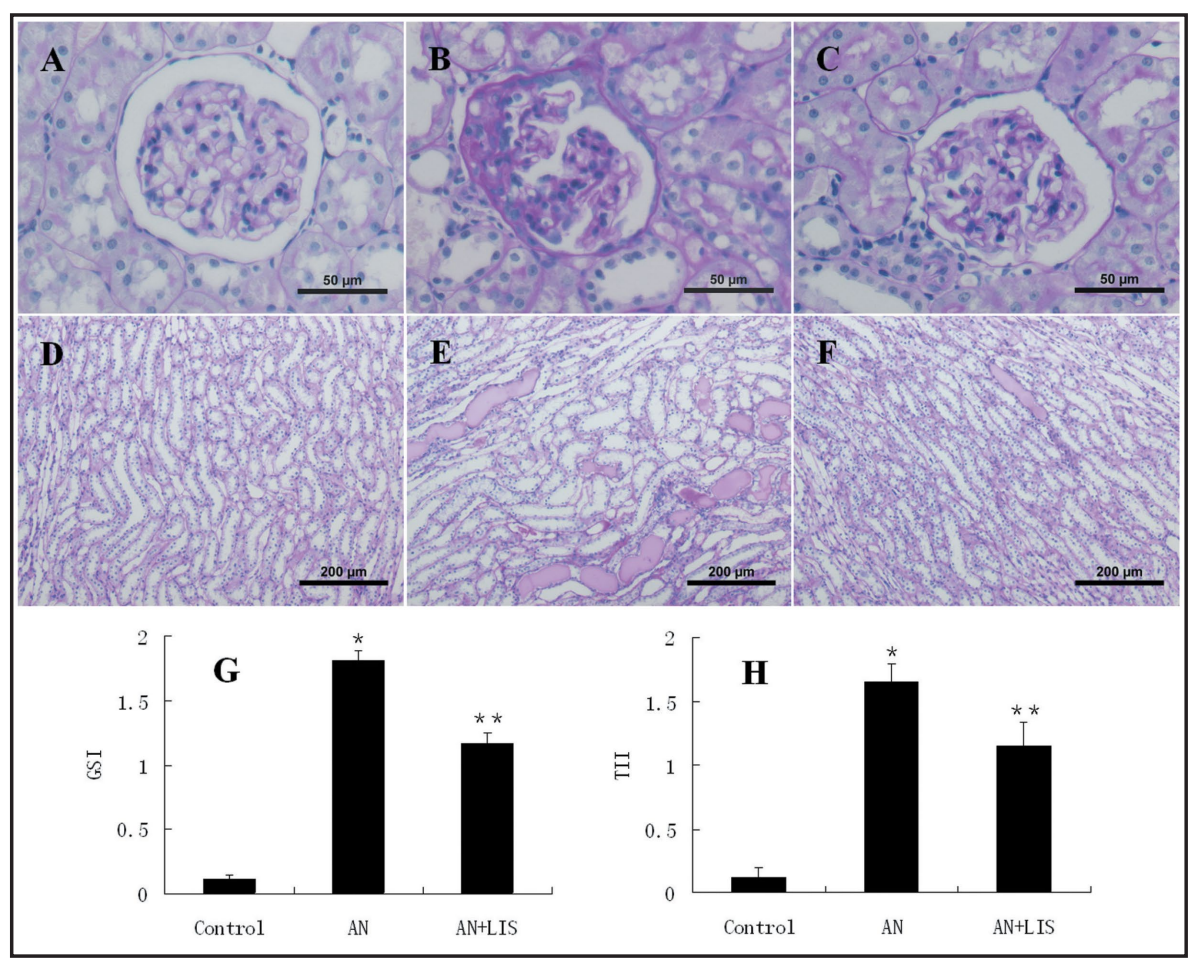

group. (A, D)

Control; (B, E) AN group; (C, F) AN+LIS group; (G, H) Bar graph showing quantitative analysis of GSI and TII. ${ }^{*} P<0.05$ when compared to control group. ${ }^{* *} P<0.05$ when compared to AN group.

Fig. 2. Renalase protein expression in kidney is reduced in model group rats compared to controls, whereas lisinopril administration increased its expression. Renalase protein expression was demonstrated immunohistochemically in renal cortex (AC) and tubule in medulla (D-E). A, D: control. B, E: AN group.

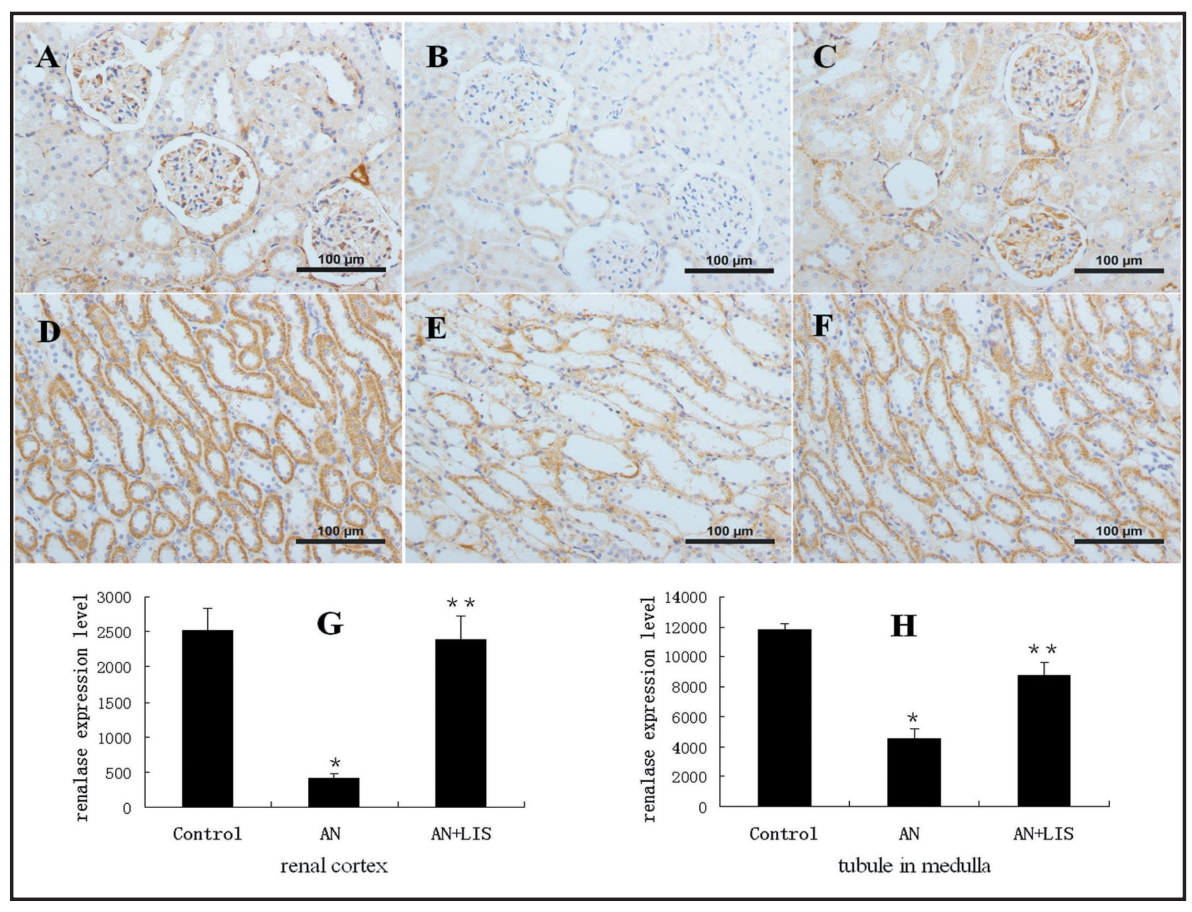
C, F: AN+LIS group. G, H: Bar graph showed renalase quantification in renal cortex and tubule in medulla for the different groups. $\mathrm{n}=6$ per group. ${ }^{*} P<0.05$ when compared to control group. ${ }^{* *} P<0.05$ when compared with the AN group. 


\section{Kidney Blood Pressure Research}

Fig. 3. Western blot analysis of renalase protein in kidney. (A) Representative immunoblot results obtained from control, AN, and AN+LIS groups are shown. (B) Densitometric analysis of western blots for renalase protein in extracts prepared from six rats per group. ${ }^{*} P<0.05$ when compared to control group. ${ }^{* *} P$ $<0.05$ when compared to the AN group.
Kidney Blood Press Res 2013;37:295-304

\begin{tabular}{l|l}
\hline DOI: 10.1159/000350157 & (c) 2013 S. Karger AG, Basel
\end{tabular}

Published onIIne: September 05, 2013

www.karger.com/kbr

Han/Sun/Xu/Zeng/Yi/Wu/Shao/Li/Yi: Renalase in Adriamycin Nephropathy

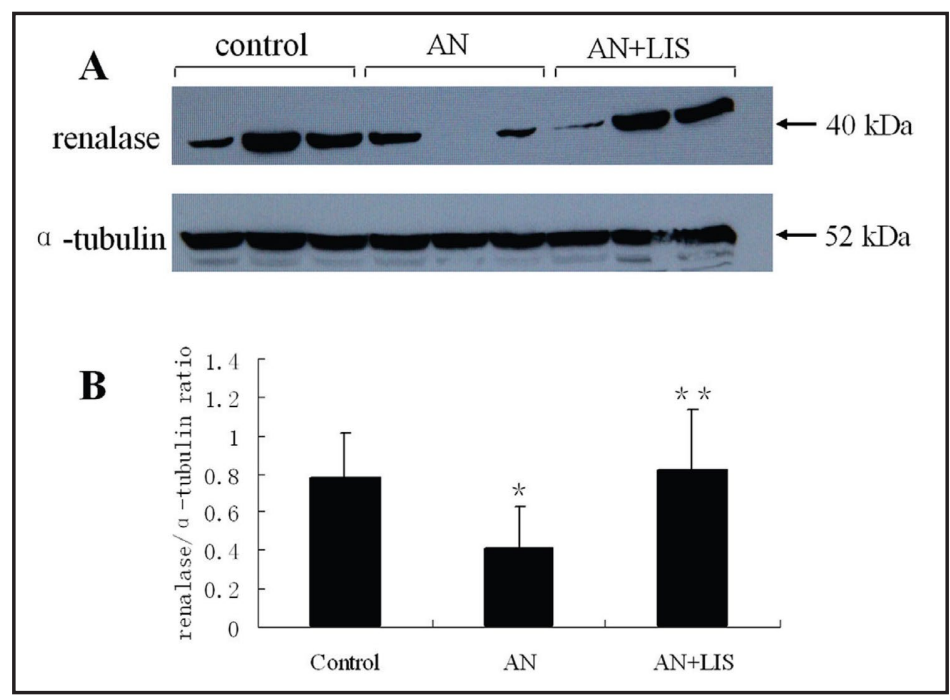

Fig. 4. (A) Serum renalase concentration was increased in both model and treatment groups. (B) 24 h urinary renalase excretion was calculated by multiplying renalase concentration by
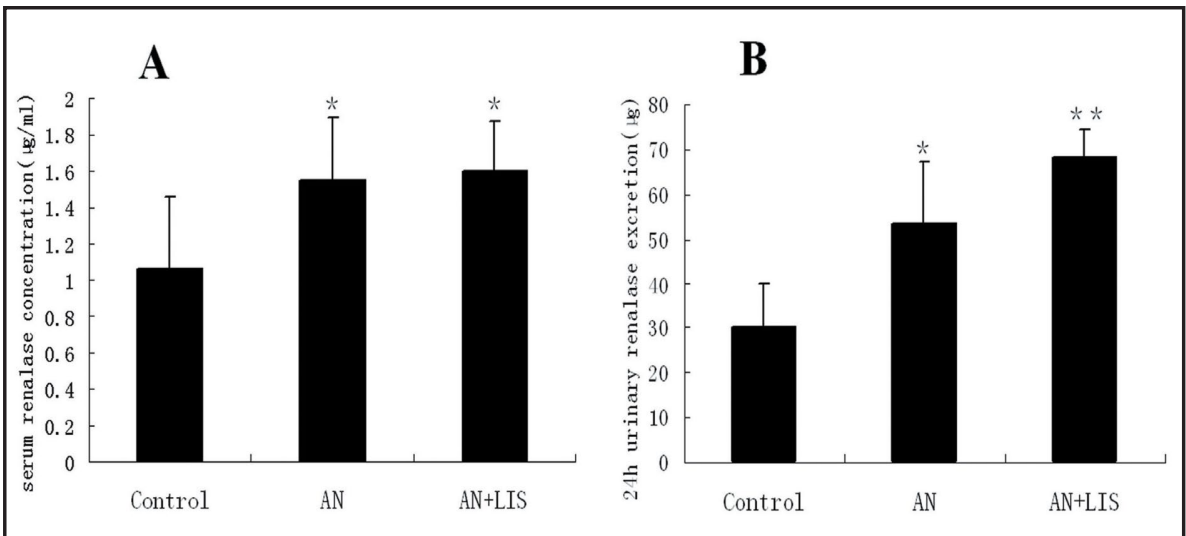
urine volume.

Urinary renalase excretion was increased in both model and treatment groups. $\mathrm{n}=6$ per group. ${ }^{*} P<0.05$ compared to control group. ${ }^{* *} P<0.05$ compared to the AN group.

group, less renalase was expressed in the proximal tubules and glomeruli than in the control group. Compared with the model group, the expression of renalase in the proximal tubules and glomeruli was significantly increased in the treatment group (Figure 2C, F). Quantitative immunohistochemical analysis demonstrated the above changes of renalase protein expression in renal tissue of rats in the three groups (Figure $2 \mathrm{G}$ and $\mathrm{H}$ ). Western blot results also showed that lisinopril improved the adriamycin-induced reduction of renalase expression in renal tissues, which was consistent with the immunohistochemistry results. As shown in Figure 3, renalase protein levels in kidney were obviously decreased in the model group compared to control animals. Renalase protein levels in the treatment group were significantly higher than in the model group, and similar to those in the control group.

\section{Renalase measurements in blood and urine}

ELISA results showed that, at 12 weeks, serum renalase concentration increased in both the model and treatment groups compared to controls (Figure 4A). The $24 \mathrm{~h}$ urinary renalase excretion was also increased in the model and treatment groups. Compared to the model group, urinary renalase excretion in treatment group demonstrated more obvious, as shown in Figure 4B. 


\section{Kidney Blood Pressure Research}

\section{Discussion}

Data from this experiment demonstrate that model group rats showed marked proteinuria, elevated SBP, hypoalbuminemia and pathological renal damage at 12 weeks. Compared to control group rats, the serum concentration and urinary excretion of renalase were increased in model group rats. However, renalase protein was decreased in renal tissues, and this was confirmed by immunohistochemistry and western blot analysis. It is probable that the reduction of renalase in kidney is a result of the renal injury induced by adriamycin. As to the elevation of serum renalase concentration, it may represent a compensatory response to elevated SBP which could increase renalase activity and blood concentration. Because renalase expression in kidney is reduced, it is possible that other organs such as the heart, skeletal muscle, and small intestine are involved in the secretion of renalase into blood. There maybe other factors contribute to the elevation of serum renalase such as detection of breakdown products and cross-reaction with unrelated epitopes. However, the complex mechanisms mediating its secretion are not yet clear. With respect to the increased urinary excretion of renalase, which is in agreement with the marked proteinuria, it may be related to the impaired permeability of the glomeruli. Compared to other previous studies, the urinary renalase concentration in our experiment seems to be higher. There maybe some reasons such as different methods, cross-reaction and unidentified factors participate in the detection process.

Hypertension, sympathetic over-activity and catecholamine accumulation play key roles in the progression of chronic kidney disease. Previous studies have revealed that renalase has a close relationship with sympathetic tone, hypertension and cardiorenal disease [1921]. Our study shows that lisinopril administration can reduce glomerulosclerosis and tubulointerstitial injury in adriamycin nephropathy rats. The hypotensive effect induced by lisinopril administration was also clearly seen. Compared to model group rats, lisinopril administration induced a significant up-regulation of renalase in kidney and increased excretion into the urine, whereas the serum concentration was similar. The hypotensive effect and renal protection of lisinopril might be explained by its regulatory effect on renalase. Although lisinopril reduced the model rats SBP to a level similar to that in the control group, the serum renalase concentration was also high in the treatment group. These results implicated that blood pressure were only one factor on renalase regulation, and that there must be some other regulators involved. In this study, lisinopril administration decreased proteinuria, but increased urinary renalase excretion. In consideration of the similar serum renalase concentration between model and treatment group, we infer that lisinopril may promote the secretion of renalase into urine through some unknown mechanism.

Our study demonstrated that serum renalase concentration increased in adriamycin model rats, which were in normal renal function condition. We did not examine the possible involvement of renalase on the renoprotection of lisinopril but only evaluated the lisinoprilinduced changes in renalase levels in renal tissues and body fluids. Previous studies about renalase roles in the kidney diseases mainly focused on the relationship between the renalase level and renal function. Some studies suggested that blood renalase level is positively correlated with glomerular filtration rate and is markedly reduced in patients with CKD and end-stage renal disease (ESRD) [22-25]. In contrast, other studies revealed that serum renalase increased in parallel to the stage of CKD [26-30]. There are several possible explanations of this apparent contradiction. First, the antibody in the ELISA kit used in these studies may recognize epitopes not detected by other studies. Second, there may be extrarenal sites involved in the synthesis and secretion of renalase. Furthermore, other as-yet unidentified signals may also participate in the pathological process.

Generally, renalase is inactive at baseline and may be activated by either a modest increase in blood pressure or brief surges in plasma catecholamines [4]. The reninangiotensin system, which is closely related to hypertension, is usually activated in kidney diseases. However, little is known about the relationship between renalase regulation and the commonly used hypotensive drugs such as ACEI. In our study, a potential mechanism 


\section{Kidney \\ Blood Pressure Research}

Han/Sun/Xu/Zeng/Yi/Wu/Shao/Li/Yi: Renalase in Adriamycin Nephropathy

that links ACEI and renalase in a rat model of adriamycin nephropathy was identified. These findings may lead to novel insights into the mechanisms of blood pressure regulation and the pathogenesis of hypertension. Recent research suggested that renalase could protect against ischemic acute kidney injury by reducing renal tubular necrosis, apoptosis, and inflammation [31]. Whether or not the renoprotective effect of renalase is related to RAS is still unclear.

\section{Conclusion}

The present study has demonstrated that lisinopril treatment can attenuate kidney injury induced by adriamycin. Lisinopril has a role in renal protection and a hypotensive effect, which may partly account for its regulation of renalase. The explicit relationship between renalase and ACEI requires further studies.

\section{Conflict of Interests}

All authors of the manuscript declare that they have no conflict of interest.

\section{Acknowledgements}

This study was supported by Grant from the National Natural Science Foundation (NO: 81202818) and Shenzhen Science and Technology project (NO: 201101025) of China.

\section{References}

1 Xu J, Li G, Wang P, Velazquez H, Yao X, Li Y, Wu Y, Peixoto A, Crowley S, Desir GV: Renalase is a novel, soluble monoamine oxidase that regulates cardiac function and blood pressure. J Clin Invest 2005;115:1275-1280.

2 Desir GV: Renalase is a novel renal hormone that regulates cardiovascular function. J Am Soc Hypertens 2007;1:99-103.

3 Wang F, Xing T, Li J, Bai M, Hu R, Zhao Z, Tian S, Zhang Z, Wang N: Renalase's expression and distribution in renal tissue and cells. PLoS One 2012;7:e46442.

4 Li G, Xu J, Wang P, Velazquez H, Li Y, Wu Y, Desir GV: Catecholamines regulate the activity, secretion, and synthesis of renalase. Circulation 2008;117:1277-1282.

5 Zhao Q, Fan Z, He J, Chen S, Li H, Zhang P, Wang L, Hu D, Huang J, Qiang B, Gu D: Renalase gene is a novel susceptibility gene for essential hypertension: A two-stage association study in northern han chinese population. J Mol Med (Berl) 2007;85:877-885.

6 Farzaneh-Far R, Desir GV, Na B, Schiller NB, Whooley MA: A functional polymorphism in renalase (glu37asp) is associated with cardiac hypertrophy, dysfunction, and ischemia: Data from the heart and soul study. PLoS One 2010;5:e13496.

7 Wu Y, Xu J, Velazquez H, Wang P, Li G, Liu D, Sampaio-Maia B, Quelhas-Santos J, Russell K, Russell R, Flavell RA, Pestana M, Giordano F, Desir GV: Renalase deficiency aggravates ischemic myocardial damage. Kidney Int 2011;79:853-860.

-8 Baraka A, El Ghotny S: Cardioprotective effect of renalase in 5/6 nephrectomized rats. J Cardiovasc Pharmacol Ther 2012;17:412-416.

9 Ptinopoulou AG, Pikilidou MI, Lasaridis AN: The effect of antihypertensive drugs on chronic kidney disease: A comprehensive review. Hypertens Res 2013;36:91-101.

10 Blankestijn PJ, London G, Fliser D, Jager KJ, Lindholm B, Goldsmith D, Wiecek A, Suleymanlar G, Agarwal R, Ortiz A: Major pathways of the reno - cardiovascular link: The sympathetic and renin - angiotensin systems. Kidney Int Suppl 2011;1:13-16. 


\section{Kidney \\ Blood Pressure Research}

Han/Sun/Xu/Zeng/Yi/Wu/Shao/Li/Yi: Renalase in Adriamycin Nephropathy

11 Kramer AB, van Timmeren MM, Schuurs TA, Vaidya VS, Bonventre JV, van Goor H, Navis G: Reduction of proteinuria in adriamycin-induced nephropathy is associated with reduction of renal kidney injury molecule (kim-1) over time. Am J Physiol Renal Physiol 2009;296:F1136-1145.

12 Tang SC, Leung JC, Chan LY, Eddy AA, Lai KN: Angiotensin converting enzyme inhibitor but not angiotensin receptor blockade or statin ameliorates murine adriamycin nephropathy. Kidney Int 2008;73:288-299.

13 Kramer AB, van der Meulen EF, Hamming I, van Goor H, Navis G: Effect of combining ace inhibition with aldosterone blockade on proteinuria and renal damage in experimental nephrosis. Kidney Int 2007;71:417-424.

14 Wapstra FH, Van Goor H, Navis G, De Jong PE, De Zeeuw D: Antiproteinuric effect predicts renal protection by angiotensin-converting enzyme inhibition in rats with established adriamycin nephrosis. Clin Sci (Lond) 1996;90:393-401.

15 Scholey JW, Miller PL, Rennke HG, Meyer TW: Effect of converting enzyme inhibition on the course of adriamycin-induced nephropathy. Kidney Int 1989;36:816-822.

16 Sun HL, Sun L, Li YY, Shao MM, Cheng XY, Ge N, Lu JD, Li SM: Ace-inhibitor suppresses the apoptosis induced by endoplasmic reticulum stress in renal tubular in experimental diabetic rats. Exp Clin Endocrinol Diabetes 2009;117:336-344.

17 Sun H, Ge N, Shao M, Cheng X, Li Y, Li S, Shen J: Lumbrokinase attenuates diabetic nephropathy through regulating extracellular matrix degradation in streptozotocin-induced diabetic rats. Diabetes Res Clin Pract 2013;100:85-95.

18 Gu R, Lu W, Xie J, Bai J, Xu B: Renalase deficiency in heart failure model of rats--a potential mechanism underlying circulating norepinephrine accumulation. PLoS One 2011;6:e14633.

19 Desir GV: Renalase deficiency in chronic kidney disease, and its contribution to hypertension and cardiovascular disease. Curr Opin Nephrol Hypertens 2008;17:181-185.

-20 Milani M, Ciriello F, Baroni S, Pandini V, Canevari G, Bolognesi M, Aliverti A: Fad-binding site and nadp reactivity in human renalase: A new enzyme involved in blood pressure regulation. J Mol Biol 2011;411:463-473.

21 Desir G: Novel insights into the physiology of renalase and its role in hypertension and heart disease. Pediatr Nephrol 2012;27:719-725.

22 Desir GV: Regulation of blood pressure and cardiovascular function by renalase. Kidney Int 2009;76:366370.

23 Wang F, Wang N-s, Xing T, Cao Y, Xiang H-y: The cloning and expression of renalase and the preparation of its monoclonal antibody. J Shanghai Jiaotong Univ (Sci) 2009;14:376-379.

24 Desir GV, Wang L, Peixoto AJ: Human renalase: A review of its biology, function, and implications for hypertension. J Am Soc Hypertens 2012;6:417-426.

25 Quelhas-Santos J, Sampaio-Maia B, Simoes-Silva L, Serrao P, Fernandes-Cerqueira C, Soares-Silva I, Pestana M: Sodium-dependent modulation of systemic and urinary renalase expression and activity in the rat remnant kidney. J Hypertens 2013;31:543-553.

-26 Malyszko J, Zbroch E, Malyszko JS, Koc-Zorawska E, Mysliwiec M: Renalase, a novel regulator of blood pressure, is predicted by kidney function in renal transplant recipients. Transplant Proc 2011;43:30043007.

-27 Przybylowski P, Malyszko J, Kozlowska S, Koc-Zorawska E, Mysliwiec M: Serum renalase depends on kidney function but not on blood pressure in heart transplant recipients. Transplant Proc 2011;43:3888-3891.

28 Malyszko J, Koc-Zorawska E, Malyszko JS, Kozminski P, Zbroch E, Mysliwiec M: Renalase, stroke, and hypertension in hemodialyzed patients. Ren Fail 2012;34:727-731.

29 Zbroch E, Malyszko J, Koc-Zorawska E, Mysliwiec M: Renalase in peritoneal dialysis patients is not related to blood pressure, but to dialysis vintage. Perit Dial Int 2012;32:348-351.

30 Zbroch E, Malyszko J, Malyszko JS, Koc-Zorawska E, Mysliwiec M: Renalase, a novel enzyme involved in blood pressure regulation, is related to kidney function but not to blood pressure in hemodialysis patients. Kidney Blood Press Res 2012;35:395-399.

-31 Lee HT, Kim JY, Kim M, Wang P, Tang L, Baroni S, D'Agati VD, Desir GV: Renalase protects against ischemic aki. J Am Soc Nephrol 2013;24:445-455. 